\title{
Utility Analysis of Simple FEC Schemes for VoIP
}

\author{
Parijat Dube ${ }^{1}$ and Eitan Altman ${ }^{1,2}$ \\ 1 INRIA, B.P.93, 06902 Sophia-Antipolis, France.\{pdube, altman\}@sophia.inria.fr \\ 2 C.E.S.I.M.O., Facultad de Ingeneria, Universidad de Los Andes, Mérida, Venezuela
}

\begin{abstract}
Forward Error Correction (FEC) is an attractive solution for recovering from packet losses in real-time applications. However, in many such applications, due to delay constraints, complex FEC schemes that may enable to approach the Shannon channel capacity cannot be used. In those cases, much simpler FEC schemes are implemented, in which a compressed copy of a packet is concatenated to some subsequent packet. In this paper we analyze the utility gained by such schemes in the case where losses are due to queueing congestion in the network. Loss probabilities in presence of FEC are obtained using sophisticated tools based on Ballot theorems. Our explicit expressions allows us to study numerically several approaches for adding FEC.
\end{abstract}

\section{Introduction}

Forward Error Correction (FEC) is considered to be an attractive solution for recovering from packet losses in real-time applications (over high speed networks [8[12]) without increasing latency. Though there are different proposals for FEC schemes, in this paper we shall focus on a simple FEC scheme that has been proposed [4] in the IETF (Internet Engineering Task Force) and implemented in audio tools like Freephone [1] and Rat [15. The scheme consists in adding a redundant copy of the original packet to the tail of the subsequent packet. If a packet is lost in the network then it can be reconstructed from its redundant copy (if it is correctly received). It was suggested to use PCM coding for data in the original packet and a low bit rate coding (like LPC, GSM, or 2-bit ADPCM) for the redundant information. Proposals to enhance the performance of this simple FEC scheme include: (i) increasing the offset $\phi_{0}$ between the original packet and its redundant copy [109] at the codec, (ii) adding multiple redundant copies of a packet in multiple subsequent ones [10, (iii) adding to a packet a redundant copy computed from a block of some preceding packets [5] etc.

Let the offset between the original packet and its redundant copy at the bottleneck be $\phi$. In a recent work [6], treating $\phi$ as a parameter it has been shown via a queueing analysis that the simple FEC scheme may not lead to an improvement in audio quality. The assumptions made in 6] include: (i) a single bottleneck link with dedicated buffer for audio flows implementing FEC and (ii) a linear utility function (A utility function is an indication of the variation

E. Gregori et al. (Eds.): NETWORKING 2002, LNCS 2345, pp. 226-239 2002.

(C) Springer-Verlag Berlin Heidelberg 2002 
of the audio quality at the receiver as a function of the transmission rate). The authors showed that for a buffer size $K$, the ratio of the volume of the redundant information to the volume of the original information $\alpha$, a Poisson arrival of packets, exponential distribution of service times and for $\phi$ less than the scaled buffer size (in terms of number of packets) $K_{\alpha}=\frac{K}{(1+\alpha)}$, adding FEC according to the simple FEC scheme always leads to a deterioration in quality caused by increased load in the network due to redundant data. They further showed that the same is also true for the limiting case $\phi \rightarrow \infty$, which forms an upper bound on the audio quality.

In their subsequent work 7], the authors showed that improvement in quality is possible with the simple FEC scheme when: (i) the (total) rate of flow(s) adding FEC is small compared to the total rate of the other flow(s), called exogenous flows not adding FEC and sharing the same bottleneck and (ii) with particular non-linear utility functions. It was also concluded that the audio quality is always an increasing function of the offset $\phi$ between the original packet and its copy. They also provided lower and upper bounds on the audio quality for case (i) with Poisson arrivals of packets but with exponential distribution of packet sizes for exogenous flows and deterministic packet sizes for audio flows implementing FEC.

It was argued in [6] that $\phi>K_{\alpha}$ may not be interesting as it could lead to unacceptable delays at the receiver. But it should be noticed that the actual offset between the original packet and its redundant copy at the bottleneck could become larger than the offset generated at the transmission codec due to multiplexing of different flows (either implementing FEC or not) at the bottleneck 1 . Moreover, in the case of large bandwidth, when many connections can be supported, a large offset does not generate necessarily a large delay, as the transmission time of a packet at the bottleneck is still very short.

In this paper we first complement the work in [6] and compute the performance for a fixed $\phi>K_{\alpha}$. It turns out that this case requires much more sophisticated computations than those in [6]. Using these results, we then compute the performances for the case of random offset obtained in situations where several flows are multiplexed. In particular, we obtain expressions for the expected audio quality for general utility functions. We use these results for an extensive numerical investigation using six different utility functions.

In this paper we deal with a Markovian framework: Poisson arrivals of packets and exponential distribution of all packet lengths and with the simple FEC scheme with $\phi_{0}=1$. We derive expressions for the quality function of an audio flow implementing FEC, multiplexed with: (i) other audio flows implementing FEC and/or (ii) exogenous flows not implementing FEC and different utility functions and $\Phi$ varying from 1 to $\infty$. These calculations will finally help us in the evaluation of the expected value of the quality function and the (possible) gain achievable with FEC in a more realistic framework of multiplexing of different flows.

\footnotetext{
${ }^{1}$ In presence of other multiplexed traffic, the offset $\phi$ is not deterministic anymore: it becomes a random variable $\Phi$, taking values from $\phi_{0}$ (the offset generated at the codec) to $\infty$.
} 


\section{Model}

In this section we shall use a simple (same as in [6]) $M / M / 1 / K$ queue to model the network and thus the loss process of audio packets. In other words, we assume that packets arrive at the bottleneck 2 according to a Poisson process with intensity $\lambda$ and the lengths of the packets are exponentially distributed with parameter $\mu$. Let $\rho=\lambda / \mu$, be the intensity of the audio traffic and with $\rho<1$, the loss probability of a packet in steady state is given by (see [11), $\pi_{\rho, K}=\frac{1-\rho}{1-\rho^{K+1}} \rho^{K}$, and for $\rho=1$ it is equal to, $\pi_{\rho, K}=\frac{1}{K+1}$.

Let $U(\alpha)$ be the utility function associated with receiving only a compressed version of a packet with a redundancy factor $\alpha, 0 \leq \alpha \leq 1$. We assume that $U(\alpha)$ is non-decreasing in $\alpha$ and $\lim _{\alpha \rightarrow 0} U(\alpha)=0$. Let $Y_{n} 3$ be a random variable defined as,

$$
Y_{n}=\left\{\begin{array}{l}
0 \quad \text { if packet } n \text { is lost } \\
1 \text { if packet } n \text { is correctly received }
\end{array}\right.
$$

\subsection{Case I: All Flows Implementing FEC}

We consider the case when an audio flow implementing FEC (we shall refer to this flow as the tagged flow) shares the bottleneck with other audio flows also implementing same type of FEC and arriving as independent Poisson processes. Let $\lambda_{a}$ be the total arrival rate of all other audio flows at the bottleneck. For each flow, the redundant information of a packet is located in the next. Thus $j+1$ packet of an audio flow will contain in addition to its own useful information some redundant information of the $j$ th packet of the same flow. Let $\bar{\rho}=\left(\lambda+\lambda_{a}\right) / \mu$. We shall consider two scenarios concerning the influence of redundancy on the packet size.

- Constant packet size model: We assume that the size of a packet is not affected by adding redundancy and thus redundancy is a overhead. The more the redundancy the less is the useful information carried by the packet.

- Constant amount of useful information: We assume that the packet size increases when adding redundancy (and thus increases with $\alpha$ ) so that the amount of useful information contained in a packet is unchanged. Increasing packet size has an impact both on the service times, and on the number of packets that can be stored at buffers. To account for this we assume [6]:

- the service time is exponentially distributed with parameter $\mu_{\alpha}=\frac{\mu}{1+\alpha}$. Thus $\bar{\rho}_{\alpha}=\bar{\rho}(1+\alpha)$.

- The amount of buffering is diminished by $1+\alpha$. Thus the scaled buffer size is $K_{\alpha}=\left\lfloor\frac{K}{1+\alpha}\right\rfloor$

\footnotetext{
${ }^{2}$ Most of the losses from a flow occur in the router having the smallest available bandwidth in the chain of routers, so that one may model the whole chain of routers by one single router called the bottleneck. This assumption has both theoretical and experimental 14/2 justification.

${ }^{3}$ subscript $n$ is for the $n$th packet of the total flow at the queue
} 
The loss probability in the presence of redundancy for this case is $\pi_{\bar{\rho}_{\alpha}, K_{\alpha}}=$ $\frac{1-\bar{\rho}_{\alpha}}{1-\left(\bar{\rho}_{\alpha}\right)^{K_{\alpha}+1}}\left(\bar{\rho}_{\alpha}\right)^{K_{\alpha}}$.

In the following analysis we shall denote by $P_{\alpha}($.$) , the probabilities evaluated at$ $\mu_{\alpha}$ and $K_{\alpha}$. Observe that by taking $\alpha=0$ in $P_{\alpha}($.$) and \pi_{\bar{\rho}_{\alpha}, K_{\alpha}}$ we get the corresponding expressions for the case of constant packet size as $K_{0}=K$ and $\bar{\rho}_{0}=\bar{\rho}$. Let $P_{\alpha}\left(Y_{n+\Phi}=1, \Phi=\phi \mid Y_{n}=0\right)$ be the probability that due to multiplexing there are $\phi-1$ packets between two consecutive packets of the tagged audio flow at the bottleneck and that the $n+\phi$ th packet is received correctly given that the $n$th packet is lost. Without loss of generality we assume that packet $n$ belongs to the tagged audio flow. We define below the expected quality function $Q(\alpha)$ for the two cases.

- Constant packet size

$$
\begin{aligned}
& Q(\alpha)= \\
& \quad P_{0}\left(Y_{n}=1\right) U(1-\alpha)+U(\alpha) P_{0}\left(Y_{n}=0\right) \sum_{\phi=1}^{\infty} P_{0}\left(Y_{n+\Phi}=1, \Phi=\phi \mid Y_{n}=0\right) \\
& =U(1-\alpha)-\pi_{\bar{\rho}, K}\left(U(1-\alpha)-U(\alpha) \sum_{\phi=1}^{\infty} P_{0}\left(Y_{n+\Phi}=1, \Phi=\phi \mid Y_{n}=0\right)\right) .
\end{aligned}
$$

- Constant amount of useful information: as the amount of useful information carried by a packet remains unchanged we define

$$
\begin{aligned}
& Q(\alpha)= \\
& \quad P_{\alpha}\left(Y_{n}=1\right) U(1)+U(\alpha) P_{\alpha}\left(Y_{n}=0\right) \sum_{\phi=1}^{\infty} P_{\alpha}\left(Y_{n+\Phi}=1, \Phi=\phi \mid Y_{n}=0\right) \\
& =U(1)-\pi_{\bar{\rho}_{\alpha}, K_{\alpha}}\left(U(1)-U(\alpha) \sum_{\phi=1}^{\infty} P_{\alpha}\left(Y_{n+\Phi}=1, \Phi=\phi \mid Y_{n}=0\right)\right) .
\end{aligned}
$$

We will next find the complementary probability $P_{\alpha}\left(Y_{n+\Phi}=0, \Phi=\phi \mid Y_{n}=0\right)$. In steady state we need to evaluate the probability $P_{\alpha}\left(Y_{\Phi}=0, \Phi=\phi \mid Y_{0}=0\right)$ which in terms of the buffer level is $P_{\alpha}\left(X_{\Phi}=K_{\alpha}, \Phi=\phi \mid X_{0}=K_{\alpha}\right)$, where $X_{k}$ is the queue size as seen by an arriving $k$ th packet. We will now evaluate $P_{\alpha}\left(X_{\Phi}=K_{\alpha}, \Phi=\phi \mid X_{0}=K_{\alpha}\right)$ for $\phi=\{1,2, \ldots\}$ using results from ballot theorem.

Let $A_{0}$ be the event of the loss of the 0th packet and $A_{\phi}$ the event of the loss of the $\phi$ th packet. We consider the following cases:

- Case 1: no packet is lost in the interval between the happening of $A_{0}$ and $A_{\phi} \Rightarrow$ the first lost packet after the 0th packet is the $\phi$ th packet.

- Case 2: $i(1 \leq i \leq \phi-1)$ packets are lost between $A_{0}$ and $A_{\phi}$.

We consider the two cases separately. The main difficulty in the case when the redundancy-offset is larger than the (scaled) buffer, i.e., $\phi>K_{\alpha}$ is that paths 
with this property may cause the buffer to become empty (even several times). This makes the analysis substantially more complex than in 6].

To proceed we first consider a general path that starts when there are $K_{\alpha}$ packets in the buffer, ends with $K_{\alpha}$ packets in the buffer, contains $\eta$ events (arrivals of Poisson process with parameter $\lambda+\lambda_{a}$ and departures) and no packets are lost. Let $\zeta_{\eta}$ be the probability of such a path. We will now express $P_{\alpha}\left(X_{\Phi}=\right.$ $\left.K_{\alpha}, \Phi=\phi \mid X_{0}=K_{\alpha}\right)$ for the two cases in terms of this probability.

- Case 1: for the $\phi$ packet to be the first lost packet there must be an epoch after the arrival of the $\phi-1$ packet that the buffer is full and the next event is an arrival. Upto that epoch the number of departures must be equal to the number of arrivals. The number of arrivals upto that epoch is $\phi-1$ and so the number of departures is $\phi-1$ to yield a total number of $\eta=2(\phi-1)$ events. Thus the probability for this case is: $\zeta_{2(\phi-1)} \frac{\lambda}{\lambda+\lambda_{a}+\mu_{\alpha}}$. However we also require that all the $\phi-1$ arrivals should be from other audio flows (coming as a Poisson process with intensity $\lambda_{a}$ ). Hence the joint probability that there are $\phi-1$ packets from other flows (due to multiplexing) at the bottleneck and the $\phi$ th packet is lost is $\zeta_{2(\phi-1)} \frac{\lambda}{\lambda+\lambda_{a}+\mu_{\alpha}}\left(\frac{\lambda_{a}}{\lambda_{a}+\lambda}\right)^{\phi-1}$.

- Case 2: First we look at $i=1$. Let the lost packet be the $j$ th arrival $(1 \leq j \leq \phi-1)$. Thus there should be no loss from the instant of the loss of 0th packet till the epoch before the arrival of the $j$ th packet. Since the queue size is $K_{\alpha}$ at $A_{0}$ and since the $j$ th packet is the first to be lost, there must be an epoch after the arrival of the $j-1$ packet that the buffer is again full and the next event is an arrival. Again, upto this epoch, the number of departures must be equal to the number of departures. The number of arrivals upto that epoch is $j-1$, so the number of departures is $j-1$ yielding a total number of $\eta=2(j-1)$. Thus the probability of the path is: $\zeta_{2(j-1)} \frac{\lambda_{a}}{\lambda+\lambda_{a}+\mu_{\alpha}} \zeta_{2(\phi-j-1)} \frac{\lambda}{\lambda_{a}+\lambda+\mu_{\alpha}}$. Also, since we require that all the $\phi-1$ arrivals should be from other audio flows we have the probability of the path as $\zeta_{2(j-1)} \frac{\lambda_{a}}{\lambda+\lambda_{a}+\mu_{\alpha}} \zeta_{2(\phi-j-1)} \frac{\lambda}{\lambda_{a}+\lambda+\mu_{\alpha}}\left(\frac{\lambda_{a}}{\lambda+\lambda_{a}}\right)^{\phi-2}$ Since $j$ can take values from 1 to $\phi-1$, the total probability for this case is $\left(\frac{\lambda_{a}}{\lambda+\lambda_{a}}\right)^{\phi-2} \sum_{j=1}^{\phi-1} \zeta_{2(j-1)} \frac{\lambda_{a}}{\lambda+\lambda_{a}+\mu_{\alpha}} \zeta_{2(\phi-j-1)} \frac{\lambda}{\lambda+\lambda_{a}+\mu_{\alpha}}$.

Now consider the case when $i=2$. We will first calculate the probability of a single path with two losses before the loss of the $\phi$ packet. Let the first loss be of $j_{1}$ and second of $j_{2}$ packet $\left(j_{1}<j_{2}, 1 \leq j_{1} \leq \phi-2,2 \leq\right.$ $\left.j_{2} \leq \phi-1\right)$. By previous arguments the probability of such a path is: $\zeta_{2\left(j_{1}-1\right)} \zeta_{2\left(j_{2}-j_{1}-1\right)}\left(\frac{\lambda_{a}}{\lambda+\lambda_{a}+\mu_{\alpha}}\right)^{2} \zeta_{2\left(\phi-j_{2}-1\right)} \frac{\lambda}{\lambda+\lambda_{a}+\mu_{\alpha}}$. The total probability for this case is (taking into consideration the requirement that all the $\phi-1$ packets should be from other audio flows):

$$
\left(\frac{\lambda_{a}}{\lambda+\lambda_{a}}\right)^{\phi-3} \sum_{j_{1}=1}^{\phi-2} \sum_{j_{2}=j_{1}+1}^{\phi-1} \zeta_{2\left(j_{1}-1\right)} \zeta_{2\left(j_{2}-j_{1}-1\right)} \zeta_{2\left(\phi-j_{2}-1\right)} \frac{\lambda \lambda_{a}}{\left(\lambda+\lambda_{a}+\mu_{\alpha}\right)^{3}}
$$


In general for $i=k(1 \leq k \leq \phi-1)$, we can write the probability as:

$$
\begin{aligned}
& \left(\frac{\lambda_{a}}{\lambda+\lambda_{a}}\right)^{\phi-(k+1)} \sum_{j_{1}=1}^{\phi-k} \sum_{j_{2}=j_{1}+1}^{\phi-k+1} \ldots \sum_{j_{k}=j_{k-1}+1}^{\phi-1} \zeta_{2\left(j_{1}-1\right)} \zeta_{2\left(j_{2}-j_{1}-1\right)} \cdots \\
& \ldots \zeta_{2\left(j_{k}-j_{k-1}-1\right)} \zeta_{2\left(\phi-j_{k}-1\right)}\left(\frac{\lambda_{a}}{\lambda+\lambda_{a}+\mu_{\alpha}}\right)^{k} \frac{\lambda}{\lambda+\lambda_{a}+\mu_{\alpha}} .
\end{aligned}
$$

Thus to complete the analysis we need to evaluate the quantity $\zeta_{\eta}$ where $\eta=2,4,6, \ldots$. To evaluate this we use the result of [13] which is based on ballot theorems. In this paper the authors have obtained the following general expression for $\zeta_{\eta}(\iota, v)$, i.e., the probability of a path that starts with $\iota\left(1 \leq \iota \leq K_{\alpha}\right)$ packets in the buffer ends with $v\left(1 \leq v \leq K_{\alpha}\right)$ packets in the buffer and contains $\eta(0 \leq \eta \leq 2 \phi+\iota-v)$ events (arrivals and departures) and no packets are lost:

$$
\zeta_{\eta}(\iota, v)=\xi_{\eta}(\iota, v)+\sum_{r=1}^{\mathcal{R}} W_{\iota} Y^{r-1}[Z(\iota, v)]
$$

where $\xi_{\eta}(\iota, v)$ is the probability of a path that starts with $\iota$ packets in the buffer ends with $v$ packets in the buffer and contains $\eta$ events $(|\iota-v| \leq \eta \leq 2 n+\iota-v$, $\left.1 \leq \iota \leq K_{\alpha}, 1 \leq v \leq K_{\alpha}\right)$ and the buffer never empties along this path, $\mathcal{R}=1+\frac{\eta-\iota-v}{2}, W_{\iota}$ and $Z$ are $\mathcal{R}$-length row and $Y$ is an $\mathcal{R} X \mathcal{R}$ matrix defined as

$$
\begin{aligned}
W_{\iota}= & \left(\xi_{\iota}(\iota, 0), \xi_{\iota+2}(\iota, 0), \ldots, \xi_{\iota+2(\mathcal{R}-1)}(\iota, 0)\right), \\
Z(\iota, v)= & \left(\xi_{\eta-\iota}(0, v), \xi_{\eta-\iota-2}(0, v), \ldots, \xi_{\eta-\iota-2(\mathcal{R}-1)}(0, v)\right), \\
Y= & \left(\begin{array}{ccccc}
0 & \xi_{2}(0,0) & \xi_{4}(0,0) & \ldots & \xi_{2(\mathcal{R}-1)}(0,0) \\
0 & 0 & \xi_{2}(0,0) & \ldots & \xi_{2(\mathcal{R}-2)}(0,0) \\
\vdots & \vdots & \vdots & \vdots & \vdots \\
\vdots & \vdots & \vdots & \vdots & \vdots \\
\vdots & \vdots & \vdots & \vdots & \vdots \\
0 & 0 & 0 & \ldots & \xi_{2}(0,0) \\
0 & 0 & 0 & \ldots & 0
\end{array}\right)
\end{aligned}
$$

For our analysis we need $\zeta_{\eta}\left(=\zeta_{\eta}\left(K_{\alpha}, K_{\alpha}\right)\right)$. Thus we get,

$$
\zeta_{\eta}\left(K_{\alpha}, K_{\alpha}\right)=\zeta_{\eta}=\xi_{\eta}\left(K_{\alpha}, K_{\alpha}\right)+\sum_{r=1}^{\mathcal{R}} W_{K_{\alpha}} Y^{r-1} Z\left(K_{\alpha}, K_{\alpha}\right)
$$

where $\mathcal{R}=1+\frac{\eta-2 K_{\alpha}}{2}=1+\frac{\eta}{2}-K_{\alpha}$. The steps for evaluation of $\xi_{\eta}(\iota, v)$ are available in 13 . Thus we can write $P_{\alpha}\left(X_{\Phi}=K_{\alpha}, \Phi=\phi \mid X_{0}=K_{\alpha}\right)$ as

$$
\begin{aligned}
& P_{\alpha}\left(X_{\Phi}=K_{\alpha}, \Phi=\phi \mid X_{0}=K_{\alpha}\right)= \\
& \quad \zeta_{2(\phi-1)} \frac{\lambda}{\lambda_{a}+\lambda+\mu_{\alpha}}\left(\frac{\lambda_{a}}{\lambda+\lambda_{a}}\right)^{\phi-1}+\frac{\lambda}{\lambda_{a}+\lambda+\mu_{\alpha}}
\end{aligned}
$$




$$
\begin{aligned}
& \sum_{k=1}^{k=\phi-1}\left[\sum_{j_{1}=1}^{\phi-k} \sum_{j_{2}=j_{1}+1}^{\phi-k+1} \ldots \ldots \sum_{j_{k}=j_{k-1}+1}^{\phi-1} \zeta_{2\left(j_{1}-1\right)} \zeta_{2\left(j_{2}-j_{1}-1\right)} \ldots\right. \\
& \left.\ldots \zeta_{2\left(j_{k}-j_{k-1}-1\right)} \zeta_{2\left(\phi-j_{k}-1\right)}\left(\frac{\lambda_{a}}{\lambda_{a}+\lambda+\mu_{\alpha}}\right)^{k}\left(\frac{\lambda_{a}}{\lambda+\lambda_{a}}\right)^{\phi-(k+1)}\right] .
\end{aligned}
$$

Thus knowing $P_{\alpha}\left(X_{\Phi}=K_{\alpha}, \Phi=\phi \mid X_{0}=K_{\alpha}\right)$ we have $P_{\alpha}\left(Y_{\Phi}=0, \Phi=\phi \mid Y_{0}=\right.$ $0)$ and thus we have $P_{\alpha}\left(Y_{\Phi}=1, \Phi=\phi \mid Y_{0}=0\right)=P_{\alpha}\left(\Phi=\phi \mid Y_{n}=0\right)-P_{\alpha}\left(Y_{\Phi}=\right.$ $\left.0, \Phi=\phi \mid Y_{0}=0\right)=P_{\alpha}(\Phi=\phi)-P_{\alpha}\left(Y_{\Phi}=0, \Phi=\phi \mid Y_{0}=0,1 \leq \phi<\infty\right.$. We next find the distribution of $\Phi$. Observe that $\Phi-1$ is the number of packets of other flows (other audio flows arriving as an independent Poisson process with parameter $\left.\lambda_{a}\right)$. Thus $\Phi$ is geometrically distributed: $P_{\alpha}(\Phi=n)=\gamma^{n-1}(1-\gamma)$ for $n \geq 1$, with $\gamma=\gamma_{1}=\frac{\lambda_{a}}{\lambda+\lambda_{a}}$. Knowing this we can evaluate the expected quality function $Q(\alpha)$ using (1), (2).

\subsection{Case II: Multiplexing between Audio Flows Implementing FEC and Exogenous Flows for the Constant Packet Size Case}

The case when an audio flow implementing FEC shares the bottleneck with other audio flows also implementing the same FEC scheme and with some other exogenous flows not implementing FEC can be analysed similarly for the case of constant packet size. We model the packets of exogenous arriving as an independent (from audio flows) Poisson process with intensity $\lambda_{e}$ and hence the total arrival process is again a Poisson process with parameter $\lambda+\lambda_{a}+\lambda_{e}$. Further we assume that the distribution of the size of all packets of all flows is exponential with parameter $\mu$. With $\bar{\rho}=\frac{\lambda+\lambda_{a}+\lambda_{e}}{\mu}$, the loss probability of a packet is steady state (with $\bar{\rho}<1$ ) is $\pi_{\bar{\rho}}=\frac{1-\bar{\rho}}{1-\bar{\rho}^{K+1}} \bar{\rho}^{K}$, and for $\bar{\rho}=1$ it is equal to $\pi_{\bar{\rho}}=\frac{1}{(1+K)}$. The expressions for expected quality can be obtained from the expressions for the case without exogenous but with $\lambda_{a}$ replaced by $\lambda_{a}+\lambda_{e}$ everywhere. For the case of constant amount of useful information it is not clear how we should scale the service rate and the buffer size for this scenario.

\section{$3 \quad$ Numerical Examples with Different Utility Functions}

We will next evaluate the expected quality achieved using different utility functions. The utility functions that we consider are used in [67] and are of the form proposed in 10,16. We define: $U_{1}(\alpha)=\alpha, U_{2}(\alpha)=\sqrt{\alpha}, U_{3}(\alpha)=\alpha^{1 / 10}$, $U_{4}(\alpha)=\operatorname{esc}\left(\alpha-\alpha_{0}\right)\left(\frac{1-\cos (\pi \alpha)}{2}\right)^{1 / 10}, U_{5}(\alpha)=\left(\frac{1-\cos (\pi \alpha)}{2}\right)^{1 / 3}$ and $U_{6}(\alpha)=1$ if $\alpha>0$ otherwise $U_{6}(\alpha)=0$. All utilities are concave starting at some minimum and reach $U(\alpha)=1$ for $\alpha=1$. The least concave is the linear utility function $U_{1}$, which is thus proportional to the amount of information which is well received. The most concave function is $U_{6}$. Although it does not represent a utility of any real application, it can be used to obtain an upper bound on the gain achieved with employing FEC. Indeed, we see that $U_{6}$ is larger than any other 
utility, so it follows that in both models described by (11) and (2), it should give the best quality. The utility $U_{4}$ is zero for $\alpha \leq \alpha_{0}$. This is typical for real time applications with a minimum hard constraint. For example, the constraint may represent the fact that the throughput of existing codecs for voice applications cannot go beneath some bound. We shall plot the quality (expected) function with these six utility functions under different scenarios. For the plots we denote by $M$ the actual buffer size and by $\rho$ that total load. Also note that in all the plots that follow the utility functions are represented as: $*-U_{1},+-U_{2}$, . $-U_{3}, o-U_{4},-U_{5}$ and finally $--U_{6}$.

1. A single audio flow implementing FEC traversing the bottleneck (no multiplexing): When a single audio flow traverses the bottleneck (i.e., $\lambda_{a}=\lambda_{e}=$ 0 ), then the spacing between a packet and its redundancy at the bottleneck is the same as that generated at the transmission codec. We take $\lambda=1$ and $\rho=\lambda / \mu$. We next plot the quality function with the six utility functions for this scenario and for the cases when: (i) the packet sizes (and hence the buffer sizes) remains unchanged after adding redundancy and the quality function is given by (1) in Figs. 1, 2(ii) when the packet sizes changes after adding redundancy and the quality function is given by (2) in Figs. 30
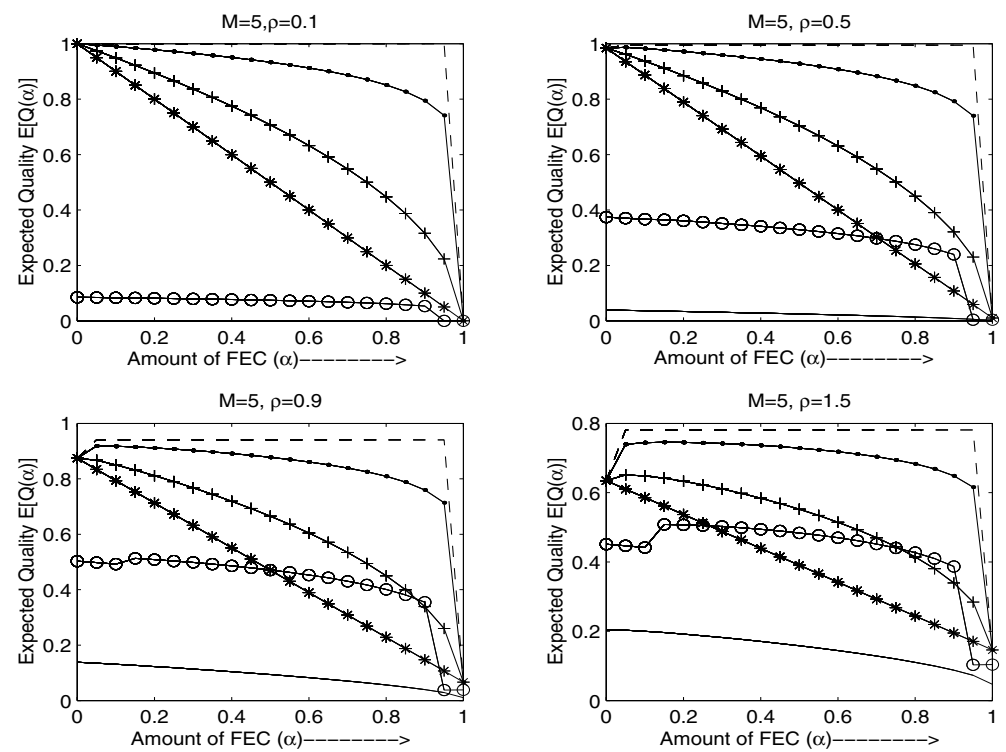

Fig. 1. A single audio flow implementing simple FEC traversing the bottleneck with quality function given by (1).

We observe that

- as expected, $U_{6}$ gives an upper bound for the quality. More generally, for two utility functions, $U_{j}$ and $U_{i}$, if $U_{i} \geq U_{j}$ for all $\alpha$, then the corre- 

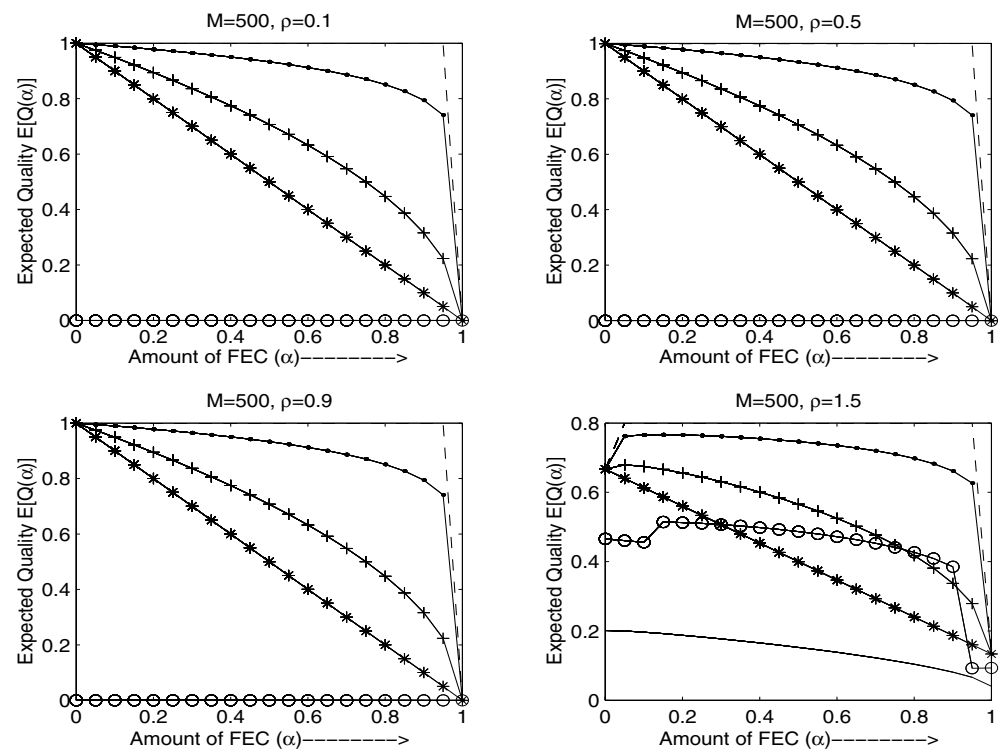

Fig. 2. A single audio flow implementing simple FEC traversing the bottleneck with quality function given by (II).
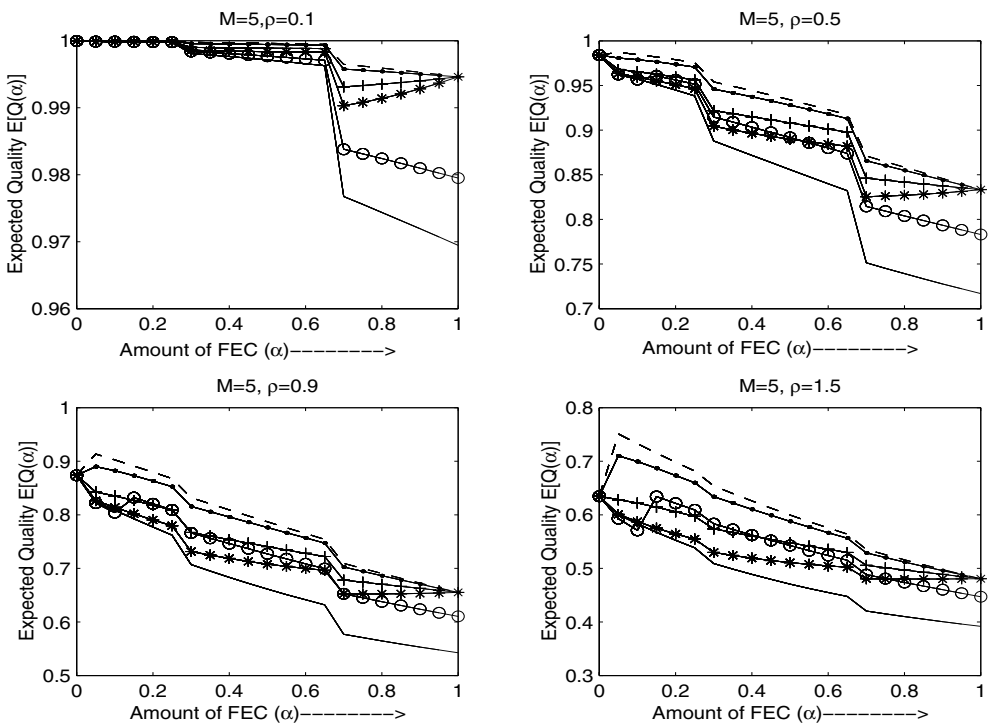

Fig. 3. A single audio flow implementing simple FEC traversing the bottleneck with quality function given by (2). 

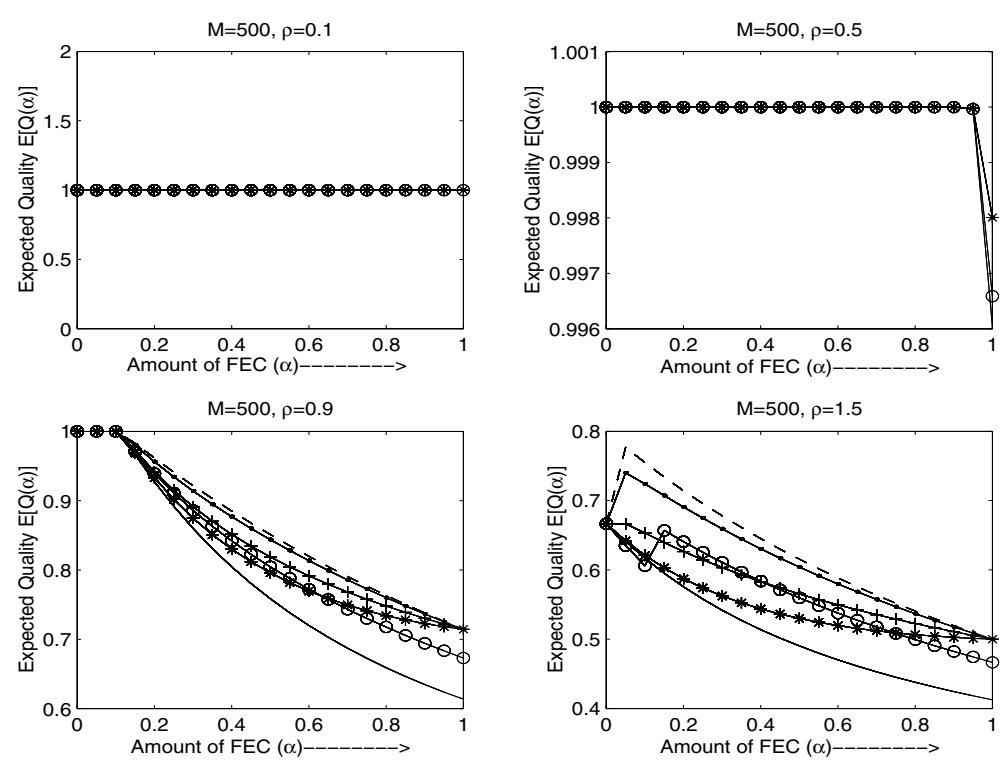

Fig. 4. A single audio flow implementing simple FEC traversing the bottleneck with quality function given by (2).

sponding quality is larger for both cases (11) and (2). This is confirmed in the figures, taking into account that $U_{6} \geq U_{3} \geq U_{2} \geq U_{1}$.

- For large buffer size $(M=500)$ the quality is almost the same for all $\rho \leq 0.9$; the reason is that the loss probabilities are very small and almost all contribution for the quality is from the utility of unlost packets.

- For $\rho>1$ we see that we gain by adding FEC (for any buffer size, and for both cases described by (11) and (2) ), for utilities $U_{3}, U_{4}, U_{6}$. For small buffer size $M=5$ we gain also for $\rho=0.9$ when using utility $U_{3}, U_{4}, U_{6}$ for both the cases but for very low $\alpha, 0.1$ or less.

- The linear utility function always decreases with FEC for any $\rho$ and any $M$.

- The quality is higher with constant information model than with constant packet size model.

2. An audio flow implementing simple FEC sharing the bottleneck with other audio flows implementing the same FEC: This is the case I (Sec. 2.1) of our analysis. We take $\lambda=0.1$ and $\lambda_{a}=0.9$ and $\rho=\left(\lambda+\lambda_{a}\right) / \mu$. We plot the expected quality function with the six utility functions for this scenario and for the cases when: (i) the packet sizes (and hence the buffer sizes) remains unchanged after adding redundancy and the quality function is given by (II) in Figs. 5, 6 (ii) when the packet sizes changes after adding redundancy and the quality function is given by 7 , Due to multiplexing $\Phi$ takes values in $\{1,2, \ldots\}$. For our numerical calculations we restricted to $\phi=\{1,2, \ldots, 8\}$ as for $\phi \geq 9$, the contribution to $Q(\alpha)$ was negligible. Thus for buffer size of 5 we will have the spacing exceeding the buffer size. Observe that the 

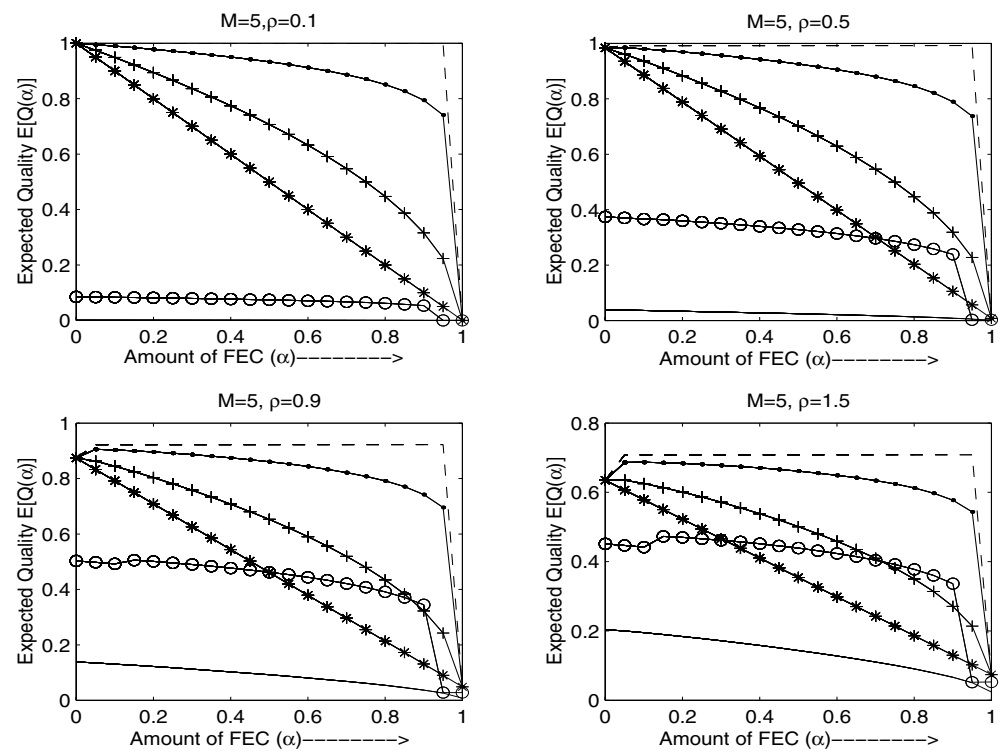

Fig. 5. An audio flow implementing simple FEC sharing the bottleneck with other audio flows implementing the same FEC scheme with quality function given by (II).
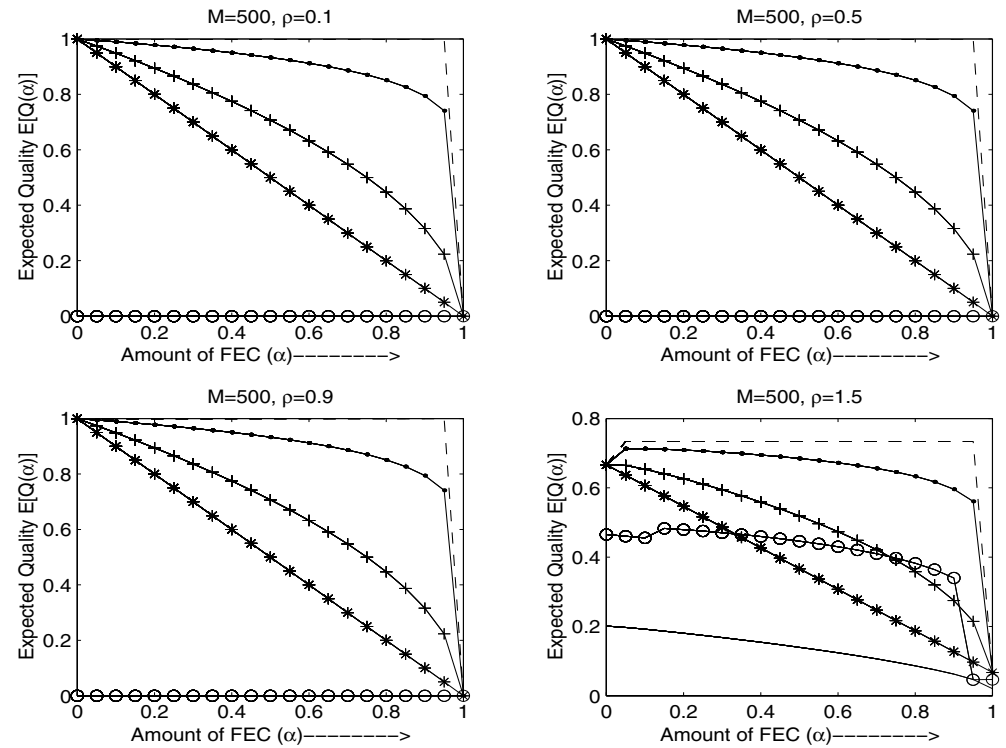

Fig. 6. An audio flow implementing simple FEC sharing the bottleneck with other audio flows implementing the same FEC scheme with quality function given by (1). 

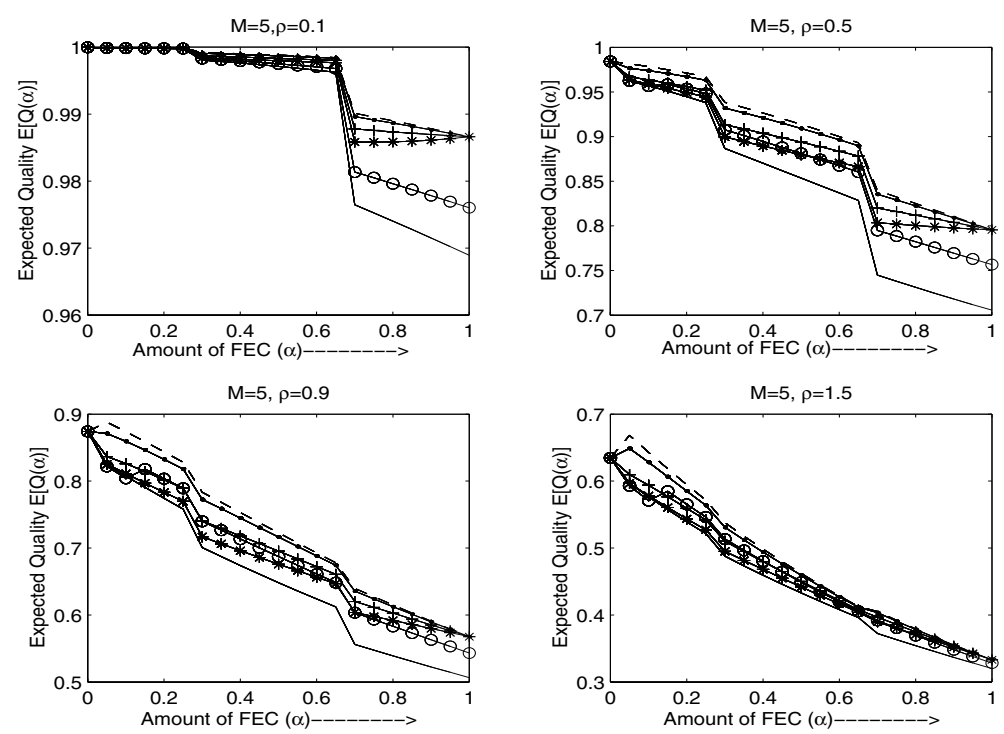

Fig. 7. An audio flow implementing simple FEC sharing the bottleneck with other audio flows implementing the same FEC scheme with quality function given by (2).
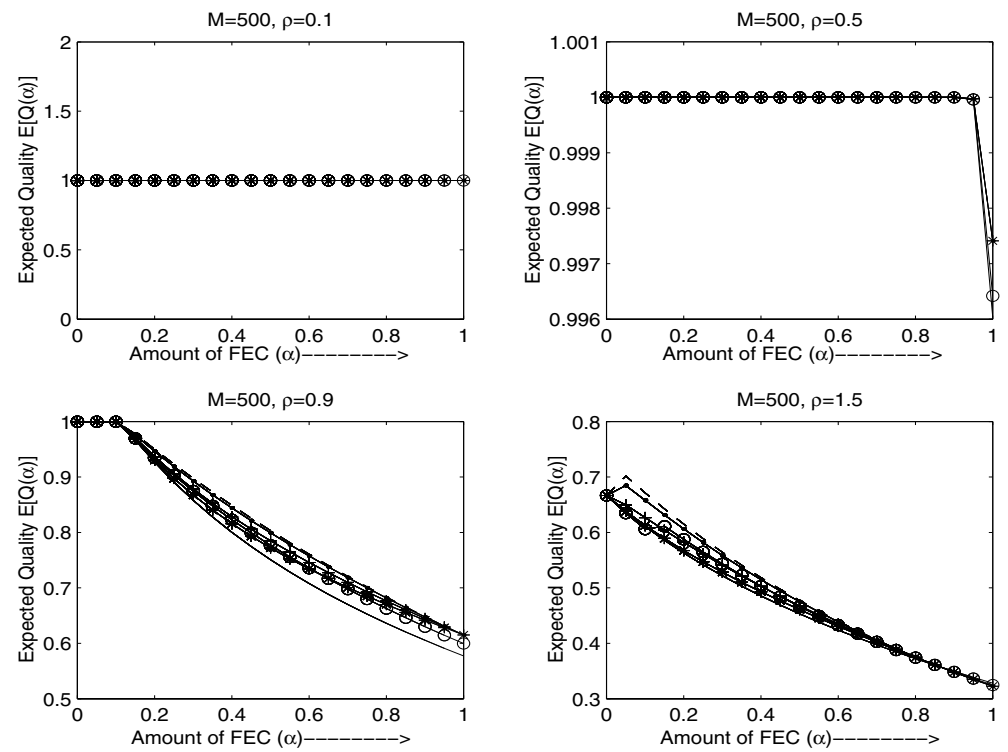

Fig. 8. An audio flow implementing simple FEC sharing the bottleneck with other audio flows implementing the same FEC scheme with quality function given by (2). 
plots (5) and (6) are almost similar to plots (11) and (2) respectively. From (7) and (8) We observe that for large $\rho(>1)$, the buffer size does not affect the expected quality.

Also from all the plots it is observed that $U_{5}$ always gives a lower bound on expected quality for large $\alpha(\geq 0.2)$.

\section{Conclusion}

In this paper we studied the (possible) gain obtained with a simple FEC scheme when the losses are due to buffer overflow at the bottleneck. We obtained the loss probabilities in the presence of FEC using ballot theorem. To this end we generalize the analysis in [6] (which was for $\phi<K_{\alpha}$ ) and computed the loss probability for a fixed $\phi$ taking values from 0 to $\infty$. Using these results we obtained the expressions for the expected audio quality for general utility functions and then utilised the tools developed for a detailed numerical studies with six utility functions under various scenarios of multiplexing at the bottleneck. Our future work is to analyse delay aware utility functions [3] and to do utility analysis of other more intelligent and efficient FEC schemes to quantize the (possible) gain.

\section{References}

1. A. V. Garcia, S. Fosse-Parisis. (FreePhone Audio Tool) High-Speed Networking Group. INRIA, Sophia Antipolis, France.

2. J. C. Bolot. End-to-End Delay and Loss Behavior in the Internet. Proc. Sigcomm '93, pages 289-298, 1993.

3. C. Boutremans, J. L. Boudec. Adative Delay Aware Error Control for Internet Telephony. Proc. IPTEL 2001, 2001.

4. C. Perkins, L. Kouvelas, O. Hodson, V. Hardman. RTP payload for redundant audio data. RFC 2198 (1997).

5. D. R. Figueiredo, E. de Souza e Silva. Efficient Mechanisms for Recovering Voice Packets in the Internet. Proc. IEEE Globecom '99, 1999.

6. E. Altman, C. Barakat and V. M. Ramos R. Queueing Analysis of Simple FEC Schemes for IP Telephony. Proc. IEEE Infocom 2001, April 2001.

7. E. Altman, C. Barakat, V. M. Ramos R. On the Utility of FEC Mechanisms for Audio Applications. Proc. Second International Workshop on Quality of Future Internet Services, Qofis 2001, 24-26 Sept., 2001, Coimbra, Portugal. See also INRIA Research Report No. RR-3998 at http://wwwsop.inria.fr/mistral/personnel/Eitan.Altman/perf.html.

8. E. W. Biersack. Performance Evaluation of FEC in ATM Networks. Proc. ACM Sigcomm '92, pages 248-257, Aug. 1992.

9. I. Kouvelas, O. Hodson, V. Hardman, J. Crowcroft. Redundancy Control in RealTime Internet Audio Conferencing. Proc. of AVSPN '97, Aberdeen, Scotland, Sept. 1997.

10. J. C. Bolot, S. Fosse-Parisis, D. Towsley. Adaptive FEC-Based Error Control for Interactive Audio in the Internet. Proc. IEEE Infocom 1999.

11. L. Kleinrock. Queueing Systems, Vol. I. John Wiley, New York, 1976. 
12. N. Shacham, P. McKenney. Packet Recovery in High-Speed Networks Using Coding and Buffer MAnagement. Proc. IEEE Infocom '90, pages 124-131, May 1990.

13. O. Gurewitz, M. Sidi, I. Cidon. The Ballot Theorem Strikes Again: Packet Loss Process Distribution. IEEE Trans. on Information Theory, 46(7):2588-2595, 2000.

14. O. J. Boxma. Sojourn Times in Cyclic Queues: the influence of the slowest server, Computer Performnace and Reliability. Elsevier Science Pubs. B. V. (NorthHolland), 1988.

15. Mice Project. (RAT: Robust Audio Tool) Multimedia Integrated Conferencing for European Researchers. University College London, U.K.

16. S. Shenker. Fundamental Design Issues for the Future Internet. IEEE Journal on Selected Areas in Communication, 13(7), September 1995. 